\title{
Chronic Childhood Stress: Psychometric Properties of the Chronic Stress Questionnaire for Children and Adolescents (CSQ-CA) in Three Independent Samples
}

\author{
Esther I. de Bruin ${ }^{1,2}$ • Dominik S. Sieh ${ }^{1,2,3}$. \\ Bonne J. H. Zijlstra ${ }^{1}$ • Anne-Marie Meijer ${ }^{1}$
}

Accepted: 5 June 2017 / Published online: 25 June 2017

(C) The Author(s) 2017. This article is an open access publication

\begin{abstract}
Stress in children and adolescents is common and related to many developmental problems. However, most studies have made no distinction between temporary or chronic stress due to the lack of a suitable questionnaire. This study tested the factor structure, reliability and validity of the 17-item self-report Chronic Stress Questionnaire for Children and Adolescents (CSQ-CA) in three samples, that is, 717 adolescents from the general population, 161 adolescents of parents with a severe chronic medical condition (CMC) like multiple sclerosis, and 113 adolescents with healthy parents. Results showed that a one-factor solution provided a reasonable fit overall. Reliability was good ( $\alpha=.80$ to .88 ). Convergent validity was supported by positive relations between total stress scores and internalizing and externalizing problem behaviors, experience of daily hassles, and maladaptive emotion regulation strategies and negative relations with quality of life, happiness, mindfulness, self-esteem, and coping skills. Adolescents from all samples, who themselves had a light CMC like asthma, showed higher chronic stress levels than those without a CMC. In line, adolescents of parents with a severe $\mathrm{CMC}$ reported more chronic stress than those of healthy parents, and adolescents with a chronic illness themselves and a parent with a chronic illness, showed the highest scores across the two family types. Overall, this study presents
\end{abstract}

Esther I. de Bruin

e.i.debruin@uva.nl

1 Research Institute of Child Development and Education (RICDE), Research Priority Area Yield, University of Amsterdam, Nieuwe Achtergracht 127, 1018 WS Amsterdam, the Netherlands

2 UvA Minds, Academic Outpatient Child and Adolescent Treatment Center, University of Amsterdam, Plantage Muidergracht 14, 1018 TV Amsterdam, the Netherlands

3 Faculty of Social and Behavioural Sciences, Institute of Psychology, Department of Clinical Psychology, Leiden University, Leiden, the Netherlands 
good psychometric properties of the first available measure of chronic stress in children and adolescents.

Keywords Chronic $\cdot$ Childhood $\cdot$ Stress

\section{Introduction}

Stress is an important determinant for child and adolescent quality of life, psychological functioning and health problems on the long term (Hocking and Lochman 2005; Pedersen and Revenson 2005; Sieh et al. 2014). With respect to the concept of stress, it is important to make distinctions about the properties of the stressor, coping with the stressor and the feelings of being stressed, and temporary or chronic stress. These different aspects of stress are reflected in questionnaires that are developed to measure child and adolescent stress. Byrne et al. (2007) developed the Adolescent Stress Questionnaire (ASQ) to estimate the nature of adolescent stressors and Compas et al. (2001) created the Responses to Stress Questionnaire (RSQ) to measure the involuntary (stress reactivity) and voluntary (coping) reactions of children and adolescents in response to stressful events. To our knowledge, the Chronic Stress Questionnaire for Children and Adolescents (CSQ-CA) presented in this study is currently the only measure worldwide that specifically assesses feelings of chronic stress in children and adolescents.

To a significant degree, stress research is based on the stress theory of Lazarus and Folkman (1984), which differentiates between primary and secondary appraisal. Primary appraisal refers to the interpretation of life events and hassles as stressful, while secondary appraisal involves that individuals evaluate their resources and ability to cope with the perceived stressor. The perception of stress depends on both types of appraisal. A negative evaluation might enhance stress for a subsequent comparable stressful event and thereby preserve or enhance feelings of stress, leading to chronic stress, which combines current, anticipatory and retrospective stress. The finding that high baseline stress levels were positively correlated to more fragmented sleep during exam times in a prospective study with adolescents (Dewald et al. 2014) supports the idea that pre-existing stress may influence behavior under subsequent stressful situations. Further, individuals suffering from chronic stress may not be able to indicate the exact cause and time for which they perceive stress (Verhaeghe et al. 2005). So, feelings of chronic stress are indications of the impact of stressors and insufficient coping responses or resources on the child due to stressful circumstances during an extended period. These feelings may develop gradually, are unacknowledged or accepted as 'normal' by the child (Dufour et al. 2006; Forehand et al. 1998; Sieh et al. 2010a; Verhaeghe et al. 2005).

Chronic childhood stress might be the result of different situations and circumstances. First, family life events (i.e., severe parental illness) occurring in childhood are important causes of stress. A longitudinal study showed that long-term stress in children was related to parental depression due to parental stroke (Sieh et al. 2010b). In another study on parental cancer, one fifth of the 
sons and more than one third of the daughters displayed high levels of stress and these children also reported higher levels of internalizing problem behaviors (Huizinga et al. 2005). Second, social circumstances such as a low socioeconomic status, or growing up in adverse physical and psychosocial conditions are related to childhood stress. Children in these circumstances showed elevated levels of resting blood pressure and overnight neuroendocrine indices of hypothalamic pituitary activity indicating heightened stress (Evans and English 2002). And third, peer relationships or school circumstances might be a cause of childhood stress. For instance academic difficulties, or being bullied by peers may contribute to feelings of stress (Williamson et al. 2003). For chronically stressed children and adolescents, help in an early stage may prevent developmental and health problems. So, a reliable and valid questionnaire is highly needed to trace these children.

This study investigates the psychometric properties of the Chronic Stress Questionnaire for Children and Adolescents (CSQ-CA). To this aim, we examined the factor structure, reliability, convergent, and divergent validity of the CSQ-CA in three different adolescent samples (general population, and adolescents of chronically ill and healthy parents). Several questionnaires, measuring daily functioning, problem behaviour, and coping strategies are used to test the convergent and divergent validity. To ascertain that the CSQ-CA measures chronic stress (Hartong et al. 2003), we compared adolescents of parents with a chronic medical condition (CMC) with adolescents from the general population and adolescents with healthy parents. This paper focuses specifically on chronic stress in adolescents, since all samples included are based on data from children between 10 and 20 years. According to guidelines of the World Health Organization adolescence is defined as the developmental stage between 10 and 20 years of age, which starts with the onset of puberty and ends with an adult identity. Sample 1 is composed by adolescents from the general population. Sample 2 consists of adolescents of parents with a chronic medical condition (CMC) such as multiple sclerosis and stroke, and Sample 3 includes adolescents with two healthy parents from non-separated families.

The following hypotheses were tested. First, we explored whether chronic stress in adolescence, as rated by the CSQ-CA, can be considered a onedimensional construct. We therefore assessed the factor structure of the CSQ$\mathrm{CA}$ in a general population of adolescents. Second, we hypothesized to find a positive relationship between the CSQ-CA and internalizing and externalizing problem behavior, the experience of daily hassles, and maladaptive emotion regulation strategies such as rumination, or catastrophizing (convergent validity). We further expected a negative relationship between the CSQ-CA and quality of life, happiness, mindfulness, self-esteem, adaptive emotion regulation strategies such as acceptance and positive reappraisal, and coping strategies such as seeking social support (divergent validity). Third, we expected adolescents who themselves suffered from a CMC to report higher scores on the CSQ-CA than those who did not suffer from a CMC, and we expected adolescents of parents with a CMC to report higher levels of chronic stress than adolescents with healthy parents. Last, we compared adolescent CSQ-CA scores across the three samples. 


\section{Study 1}

The aim of the first study was to test the hypothesized single factor structure and to explore the reliability of the CSQ-CA of adolescents from the general community. In addition, we examined correlations between the CSQ-CA and measures of happiness, quality of life, emotion regulation strategies, and mindfulness.

\subsection{Study 1 Methods}

\subsubsection{Participants and Procedure}

The first sample consisted of $n=717$ adolescents from two high schools in the Netherlands (mean age $=14.59 ; S D=1.46 ; 48 \%$ boys). Head masters gave oral and written permission for participation of their school. Parents $(n=749)$ were approached via letters and information brochures of which 24 (3.2\%) refused to participate, and a further eight $(1.1 \%)$ of the youngsters refused to fill out the questionnaires at the time of testing. Further, there were 5 students $(.7 \%)$ with missing values on CSQ-CA items. This study, as well as Study 2 and 3, was approved by the ethics committee of the Research Institute of Child Development and Education of the University of Amsterdam. The CSQ-CA and other questionnaires were administered anonymously in the classroom (approximately $30 \mathrm{~min}$ ). The adolescents also registered whether they were suffering from any forms of mild CMC's, such as asthma and headaches (which were different from the more severe CMC's in parents in Study 2). The vast majority of the adolescents were of Dutch origin (96.5\%). The mean education level of this sample was high $(38.5 \%$ mixed high school $\mathrm{HAVO} / \mathrm{VWO} /$ gymnasium; $32.1 \%$ HAVO; $29.4 \% \mathrm{VWO}$ /gymnasium). In the Netherlands, VWO/gymnasium is the highest level of high school, pre-university education (duration 6 years), and HAVO is the level below that, senior general secondary education (duration 5 years).

\subsection{Study 1 Measures}

\subsubsection{Chronic Stress Questionnaire for Children and Adolescents (CSQ-CA)}

The CSQ-CA (Hartong et al. 2003) assesses self-reported chronic stress levels in children and adolescents. Seventeen items (plus two positively stated items aimed to make the questionnaire less worrisome that are not included in the total score. Item 7 and Item 19 are therefore missing from the tables) are rated on a 4-point scale $(1=$ not true for me at all to $4=$ completely true for me $)$. Scores range from 17 to 68; a higher score indicates more stress in the past 3 months (e.g., "I feel that I have to do too many things at the same time", "I easily over react to situations", and "I often feel relaxed"). Reliability was good in a variety of samples (e.g., Meijer et al. 2008; Sieh et al. 2012). Items from the CSQ-CA were inspired by the Depression Anxiety Stress Scales (DASS). For this, we received permission from the authors of the DASS (De Beurs et al. 2001; Lovibond and Lovibond 1995; DASS-NL). 


\subsubsection{Subjective Happiness Scale (SHS)}

The SHS is a measure of happiness (Lyubomirsky and Lepper 1999) and consists of 4 items that are rated on a 7-point scale. Reliability, test-retest reliability, and divergent and convergent reliability have all shown to be good (i.e., Lyubomirsky and Lepper 1999; Tkach and Lyubomirsky 2006). Reliability in the current sample was acceptable $(\alpha=.74)$.

\subsubsection{Pediatric Quality of Life Inventory Scale (PedsQL)}

Quality of life was assessed with the PedsQL (Varni et al. 1999). The PedsQL is a 23item 5-point questionnaire measuring quality of life in four domains: physical, emotional, social and school functioning and has been shown to be valid and have good reliability (Varni et al. 2001). A so called PsychoSocial health summary score can be derived by summing the items of the Emotional, Social and School functioning subscales. In the current sample reliability varied from acceptable to good (Total PedsQL; $\alpha=.87$, PsychoSocial health; $\alpha=.83$, Physical; $\alpha=.77$, Emotional; $\alpha=.77$, Social; $\alpha=.78$, School; $\alpha=.70$ ).

\subsubsection{Cognitive Emotion Regulation Questionnaire (CERQ)}

The 36-items CERQ (Garnefski et al. 2002) measures nine different adaptive and maladaptive cognitive emotion regulation strategies. The strategies refer to different thoughts and ideas after experiencing a threatening or stressful event. Items are rated on a 5-point scale. Reliability for the different scales varies from acceptable to good (Garnefski et al. 2007). For the current study, reliabilities of the adaptive strategies were the following: Putting into perspective; $\alpha=.72$, Positive refocusing; $\alpha=.74$, Positive reappraisal; $\alpha=.76$, Acceptance; $\alpha=.71$, and Planning; $\alpha=.75$. Reliabilities of the maladaptive strategies were the following: Self-Blame; $\alpha=.72$, Other-blame; $\alpha=.77$, Rumination; $\alpha=.75$, and Catastrophizing; $\alpha=.73$. Reliability for CERQ Total scale was good; $\alpha=.89$.

\subsubsection{Mindful Attention Awareness Scale-Adolescent Version (MAAS-A)}

The MAAS-A (Brown et al. 2011; De Bruin et al. 2011) consists of 14 items that measure the level of mindfulness. The items are answered on a 6-point scale with higher scores indicating higher trait mindfulness, which refers to a higher state of nonjudgmental present-moment awareness. Reliability has shown to be good and the MAAS-A is sensitive to the effects of a mindfulness training (Brown et al. 2011). Cronbach's alpha in the current sample was .86 .

\subsubsection{Children's Acceptance and Mindfulness Measure (CAMM)}

The 10-items CAMM (De Bruin et al. 2013; Greco et al. 2011) is a measure of mindfulness in children and adolescents. Items are rated on a 5-point scale. The CAMM has been shown to be reliable, and mindfulness total scores correlated positively with quality of life, social skills and academic performance. Negative correlations 
were shown with somatic complaints, internalizing and externalizing problem behaviors (Greco et al. 2011). Reliability in this sample was good $(\alpha=.80)$.

\subsubsection{Statistical Analyses}

To test the assumed one-dimensionality of the latent trait underlying the CSQCA, a confirmatory factor analysis (CFA) was conducted in R (R Core Team 2012) using Latent Variable Analysis (LAVAAN) (Rosseel 2013). Because of the non-normal distributions of the responses to the items of the CSQ-CA, the Satorra-Bentler correction was used, giving robust standard errors and adjusted fit statistics. Further, reliability (Cronbach's alpha) was examined and Pearson correlations with mental health-related constructs were calculated to examine convergent and divergent validity.

\subsection{Study 1 Results}

\subsubsection{Factor Analyses}

The fit of the covariance matrix as implied by the single-factor model was reasonably close to the observed (asymptotic) covariance matrix: Root Mean Square Error of Approximation (RMSEA) $=.07,90 \%$ CI $(.07, .08)$, comparative fit index $=.83$. Though, the proposed single-factor model did not completely fit the observed (asymptotic) covariance matrix, adjusted $\chi^{2}(119)=590.30$; $p<.001$. The factor loadings for the one-dimensional CFA on the CSQ-CA items are presented in Table 1. All loadings were significantly larger than zero $(p<.001)$.

\subsubsection{Reliability}

Reliability based on 17 items was good, $\alpha=.87$. Corrected item-total correlations varied between .28 (Item 11) and .67 (Item 14). The average item scores and the CSQCA total score are presented in Table 2. On average, males scored lower on the CSQCA (33.74) than females $(36.51), t(720)=4.86 ; p<.001$, and age was related to CSQCA scores, $r=.13 ; p<.01$. However, age did not differ between males and females, $t(720)=.52, p>.05$. Education was not related to CSQ-CA scores, $F(2.72)=1.13$, $p>.05$.

\subsubsection{Convergent and Divergent Validity}

As expected the CSQ-CA total score correlated negatively with quality of life (PedsQL total), $r=-.61, p<.01$, happiness (SHS), $r=-.47, p<.01$, and mindfulness (CAMM and MAAS-A), $r=-.61, p<.01$, and $r=-.57, p<.01$ respectively. Further, a positive correlation was found with coping strategies overall (CERQ total score), $r=.13, p<.01$. As expected, positive correlations were found with maladaptive emotion regulation strategies, and negative correlations were found with adaptive emotion regulation strategies. For details of correlations with all the subscales, see Table 3. 
Table 1 Factor loadings of CSQCA items in Study $1(n=717)$, Study $2(n=161)$ and Study 3 $(n=113)$, completely standardized solution

\begin{tabular}{|c|c|c|c|}
\hline \multirow[t]{2}{*}{ Items } & \multicolumn{3}{|c|}{ Studies } \\
\hline & 1 & 2 & 3 \\
\hline 1. I am very patient. & .31 & .34 & .19 \\
\hline $\begin{array}{l}\text { 2. I feel that I often over } \\
\text { think things. }\end{array}$ & .48 & .47 & .37 \\
\hline $\begin{array}{l}\text { 3. I feel that I have to do too } \\
\text { many things at the same time. }\end{array}$ & .51 & .54 & .25 \\
\hline 4. I feel comfortable at school. & .38 & .41 & .37 \\
\hline 5. I easily overreact to situations. & .50 & .51 & .61 \\
\hline 6. I often feel rushed. & .60 & .71 & .48 \\
\hline $\begin{array}{l}\text { 8. I easily get angry over } \\
\text { small things. }\end{array}$ & .54 & .55 & .43 \\
\hline 9. I often feel relaxed. & .53 & .53 & .40 \\
\hline 10. I get upset easily. & .67 & .67 & .72 \\
\hline 11. I often feel restless. & .61 & .72 & .59 \\
\hline 12. I feel comfortable at home. & .30 & .54 & .10 \\
\hline 13. I feel that I am too busy. & .55 & .59 & .34 \\
\hline 14. I worry too much. & .73 & .63 & .69 \\
\hline $\begin{array}{l}\text { 15. I don't like being disturbed } \\
\text { when I am busy doing something. }\end{array}$ & .44 & .34 & .21 \\
\hline $\begin{array}{l}\text { 16. I often get upset about } \\
\text { things that are not important. }\end{array}$ & .67 & .58 & .63 \\
\hline 17. I easily feel nervous. & .57 & .54 & .53 \\
\hline $\begin{array}{l}\text { 18. I have enough time for the } \\
\text { things I want to do. }\end{array}$ & .43 & .53 & .30 \\
\hline
\end{tabular}

\subsection{Study 1 Conclusion}

In this first large sample of adolescents of the general population, the CSQ-CA proved to show good reliability and total scores of stress correlated negatively, as expected, with constructs such as quality of life, happiness, and mindfulness. Stress displayed negative correlations with emotion regulation strategies such as acceptance, positive refocussing, planning, positive reappraisal, and putting into perspective. As expected, positive correlations were found between stress and emotion regulation strategies such as self-blame, rumination, catastrophizing, and other-blame. The fit of the hypothesized one-factor solution was reasonable with reasonable to good item loadings on the single factor.

\section{Study 2}

The aim of the second study was to examine the reliability, convergent, and divergent validity of the CSQ-CA in an independent sample of adolescents of parents with a severe CMC. We further cross-validated the factor structure as observed in Study 1. The convergent and divergent validity were investigated by calculating correlations 
Table 2 Means (SD) of the CSQ-CA items and total score in Study 1, 2, and $3(n=722, n=161$, and $n=113$ )

\begin{tabular}{|c|c|c|c|c|c|c|}
\hline \multirow[t]{2}{*}{ Items } & \multicolumn{2}{|l|}{ Study 1} & \multicolumn{2}{|l|}{ Study 2} & \multicolumn{2}{|c|}{ Study 3} \\
\hline & $M$ & $S D$ & $M$ & $S D$ & $M$ & $S D$ \\
\hline Item $1^{\mathrm{a}}$ & 1.99 & .63 & 2.23 & .76 & 2.27 & .61 \\
\hline Item 2 & 2.68 & .84 & 2.60 & .99 & 2.46 & .85 \\
\hline Item3 & 2.41 & .88 & 1.97 & .83 & 2.03 & .82 \\
\hline Item $4^{\mathrm{a}}$ & 1.57 & .65 & 1.67 & .79 & 1.60 & .65 \\
\hline Item 5 & 1.93 & .82 & 1.87 & .74 & 1.85 & .79 \\
\hline Item 6 & 2.25 & .85 & 2.03 & .86 & 2.12 & .81 \\
\hline Item 8 & 1.85 & .80 & 2.02 & .79 & 1.93 & .72 \\
\hline Item $9^{a}$ & 1.91 & .77 & 2.20 & .76 & 2.00 & .67 \\
\hline Item 10 & 1.86 & .84 & 1.93 & .88 & 1.66 & .73 \\
\hline Item 11 & 1.97 & .84 & 2.04 & .90 & 1.77 & .71 \\
\hline Item $12^{\mathrm{a}}$ & 1.24 & .55 & 1.50 & .71 & 1.27 & .52 \\
\hline Item 13 & 2.56 & .94 & 2.16 & .86 & 2.15 & .82 \\
\hline Item 14 & 2.18 & .89 & 2.23 & .98 & 1.93 & .82 \\
\hline Item 15 & 2.56 & .94 & 2.59 & .86 & 2.52 & .84 \\
\hline Item 16 & 1.72 & .79 & 1.68 & .72 & 1.54 & .64 \\
\hline Item 17 & 2.18 & .87 & 2.18 & .92 & 2.06 & .76 \\
\hline Item $18^{\mathrm{a}}$ & 2.23 & .84 & 1.82 & .76 & 1.83 & .77 \\
\hline SQ-C total & 35.09 & 7.77 & 34.75 & 8.21 & 32.99 & 6.13 \\
\hline
\end{tabular}

${ }^{\text {a }}$ Reverse scored items

with constructs such as internalizing and externalizing problem behaviors, daily hassles, self-esteem and coping strategies.

\subsection{Study 2 Methods}

\subsubsection{Participants and Procedure}

Adolescents aged 10 to 20 years living at home and their parents were included. They had to have a parent with one or more CMC's for at least 6 months. Cancer was excluded because an oncological disease is not chronic by definition. Exclusion criteria for adolescents were insufficient command of Dutch, residency outside of the Netherlands, severe somatic diseases, and cognitive disabilities. Having a mild CMC like asthma was not an exclusion criterion for participants.

Participants were recruited across the Netherlands in five rehabilitation centers and several hospitals, schools, community centers, intercultural institutions, general health practitioners' offices, and public libraries. After written informed consent had been given by parents and children, several trained research assistants made an appointment to administer questionnaires at the families' homes. Adolescents received a gift voucher, a cinema ticket, or a mobile phone cover after completing the questionnaires. 
Table 3 Correlations between the CSQ-CA total scale and other constructs in a sample of adolescents from the general population ( $n=722$, Study 1)

CAMM Children's Acceptance and Mindfulness Measure, $C E R Q$ Cognitive Emotion Regulation Questionnaire, $M A A S-A$, Mindful Attention Awareness ScaleAdolescent version, PedsQL Pediatric Quality of Life Inventory Scale, SHS Subjective Happiness Scale

\begin{tabular}{ll}
\hline & CSQ-CA total \\
\hline PedsQL total & $r=-.61 ; p<.01$ \\
PedsQL PsychoSocial health & $r=-.63 ; p<.01$ \\
PedsQL physical & $r=-.37 ; p<.01$ \\
PedsQL emotional & $r=-.58 ; p<.01$ \\
PedsQL social & $r=-.37 ; p<.01$ \\
PedsQL school & $r=-.46 ; p<.01$ \\
SHS & $r=-.47 ; p<.01$ \\
CAMM & $r=-.61 ; p<.01$ \\
MAAS-A & $r=-.58 ; p<.01$ \\
CERQ total & $r=.13 ; p<.01$ \\
CERQ self-blame & $r=.35 ; p<.01$ \\
CERQ rumination & $r=.42 ; p<.01$ \\
CERQ catastrophizing & $r=.41 ; p<.01$ \\
CERQ other-blame & $r=.22 ; p<.01$ \\
CERQ acceptance & $r=-.06 ; p=.10$ \\
CERQ positive refocusing & $r=-.18 ; p<.01$ \\
CERQ planning & $r=-.09 ; p=.02$ \\
CERQ positive reappraisal & $r=-16 ; p<.01$ \\
CERQ putting into perspective & $r .01$ \\
\hline
\end{tabular}

Six participants who contacted us did not meet the inclusion criteria and six participants decided not to participate, yielding a total of 161 adolescents from 101 families. Nearly all adolescents were of Dutch origin (99.4\%). Average age was 14.58 years $(S D=2.37)$ and $52 \%$ was female.

Of the parents with CMC's, 67\% were female. Parental CMC's included multiple sclerosis $(28.2 \%)$, rheumatoid arthritis $(19.4 \%)$, brain damage $(16.5 \%)$, muscle disease $(14.6 \%)$, spinal cord injury (6.8\%), inflammatory bowel disease $(5.8 \%)$, Parkinson disease $(5.8 \%)$, and diabetes type I with physical complications $(2.9 \%)$. Mean time since diagnosis was 12.4 years and ranged between 7 months and 49 years.

\subsection{Study 2 Measures}

\subsubsection{Youth Self-Report (YSR)}

Internalizing and externalizing problem behaviors in adolescents were measured with the Youth Self-Report for which good psychometric properties have been shown (Achenbach 1991). Adolescents rated their behavioral problems on a 3point scale. Items were summed to obtain a YSR Total Problems score, as well as two broad band scales of Internalizing Problems (31 items) and Externalizing Problems (30 items). Reliabilities in the current sample were: Total Problems scale; $\alpha=.93$, Internalizing Problems; $\alpha=.91$, and Externalizing Problems; $\alpha=.81$. 


\subsubsection{Dutch Daily Hassles Questionnaire (DDHQ)}

We measured how often children perceived stressful events in their environment impacting on their personal life, using the subscale frequency of daily hassles affecting personal life of the Dutch Daily Hassles Questionnaire (Dufour et al. 2006). Personal life refers to social time with friends, school duties, and the possibility of having a job. The questionnaire contains six items and was answered on a 5-point scale. Reliability in the current sample was reasonable, $\alpha=.74$.

\subsubsection{Utrecht Coping List-Adolescent Version (UCL-A)}

Coping skills of the adolescents were measured with two 6-item scales of the Utrecht Coping List for Adolescents: Seeking Social Support and Active Problem Solving. We only used these two scales in addition to the Total Coping score because the validation study showed comparatively low reliability of the other scales (Schreurs et al. 1993). Both the Seeking Social Support, $\alpha=.89$, and the Active Problem Solving, $\alpha=.80$, subscales showed good reliability in the current sample. Cronbach's alpha for the UCL-A Total Coping score was $\alpha=.86$.

\subsubsection{School Perception Questionnaire (SPQ)}

Self-esteem was determined with the School Perception Questionnaire (8 items) with a 5 -point rating scale. The SPQ total score was related to school performance (Van der Wolf 1995). The reliability of the scale in the current sample was good, $\alpha=.89$.

\subsubsection{Statistical Analyses}

The analyses in Study 2 were in line with those reported in Study 1.

\subsection{Study 2 Results}

\subsubsection{Factor Analysis}

The RMSEA fit statistic of the covariance matrix as implied by the single-factor model was at the commonly accepted threshold for reasonable fit of .08: RMSEA $=.08,90 \%$ CI (.07, .09), indicating a less than reasonable fit, comparative fit index $=.80$. As expected, the single factor model did also not completely fit the observed (asymptotic) covariance matrix, adjusted $\chi^{2}(119)=243.20 ; p<.001$. Nevertheless, all items were significantly, $p<.001$, related to the latent scale. The item loadings are presented in Table 2.

\subsubsection{Reliability}

Reliability based on 17 items was good, $\alpha=.88$. Corrected item-total correlations varied between .32 (Item 1) and .69 (Item 11). Girls (36.53) scored significantly higher 
on the CSQ-CA than boys (33.01), $t(156)=2.60 ; p<.05$, and age was correlated with CSQ-CA scores, $r=.23 ; p<.01$.

\subsubsection{Convergent and Divergent Validity}

The CSQ-CA total score correlated positively as expected with self-reported adolescent problems (YSR Total Problems score), $r=.72, p<.01$, and with daily hassles (DDHQ), $r=.75, p<.01$. Negative correlations were found, as expected, with school-related self-esteem (SPQ), $r=-.44, p<.01$, and with coping strategies (UCL-A Total Coping), $r=-.20, p=.01$. For details of correlations with all the subscales, see further Table 4.

\subsection{Study 2 Conclusion}

In Study 2, the one-factor structure of the CSQ-CA did not fit the data well. However, this study did further support validity and reliability of the CSQ-CA in a sample of adolescents with parents with a CMC. In line with our expectations, reliability of the 17-item CSQ-CA was good. Positive correlations were found between stress and internalizing and externalizing problem behaviors, as well as between stress and the subjective experience of daily hassles. In addition, stress correlated negatively with school-related self-esteem and with coping strategies such as seeking social support, and problem solving.

\section{Study 3}

In the third study, the CSQ-CA was administered to adolescents of healthy parents who are expected to show lower stress(ors). In line with Study 2, correlations between adolescent stress and internalizing and externalizing problem behavior, daily hassles, self-esteem and coping strategies were calculated to further examine convergent and divergent validity.

\subsection{Study 3 Methods}

\subsubsection{Participants and Procedure}

Participants in Sample 3 were 113 adolescents living at home with two healthy parents from 68 families. They had to have two parents with no major CMC. Exclusion criteria for adolescents were insufficient command of Dutch, residency outside of the Netherlands, severe somatic diseases, and cognitive disabilities.

Participants were recruited across the Netherlands in schools of all types (primary education, lower and intermediate vocational education, high school, preparatory universities, and university). After written informed consent had been given by parents and children, several research assistants made an appointment to administer questionnaires at the families' homes. Adolescents received a gift voucher, a cinema ticket, or a mobile phone cover after completing the questionnaires. 
Table 4 Correlations between the CSQ-CA and other constructs in a sample of adolescents with parents with a CMC (Study 2, $n=161$ ) and parents without a CMC (Study 3, $n=113$ )

\begin{tabular}{lll}
\hline & CSQ-CA total Study 2 & CSQ-CA total Study 3 \\
\hline YSR total problems & $r=.64 ; p<.01$ & $r=.62 ; p<.01$ \\
YSR externalizing problems & $r=.39 ; p<.01$ & $r=.46 ; p<.01$ \\
YSR internalizing problems & $r=.72 ; p<.01$ & $r=.54 ; p<.01$ \\
DDHQ & $r=.75 ; p=<.01$ & $r=.14 ; p=.21$ \\
SPQ & $r=-.44 ; p<.01$ & $r=-.39 ; p<.01$ \\
UCL-A total coping & $r=-.20 ; p=.01$ & $r=-.15 ; p=.13$ \\
UCL-A seeking social support & $r=-.15 ; p=.06$ & $r=-.07 ; p=.47$ \\
UCL-A active problem solving & $r=-.19 ; p=.02$ & $r=-.17 ; p=.08$ \\
\hline
\end{tabular}

CMC Chronic Medical Condition, DDHQ Dutch Daily Hassles Questionnaire, SPQ School Perception Questionnaire, UCL-A Utrecht Coping Questionnaire-Adolescent version, YSR Youth Self Report.

Most of the adolescents were of Dutch origin (96\%). Approximately half of the sample $(52.6 \%)$ consisted of girls and the average age was 14.54 years $(S D=2.25)$.

\subsection{Study 3 Measures}

In line with Study 2, the YSR (Total Problems score: $\alpha=.87$; Internalizing Problems: $\alpha=.78$; Externalizing Problems: $\alpha=.78)$, DDHQ $(\alpha=.37)$, SPQ $(\alpha=.88)$, and UCL-A (Total Coping scale: $\alpha=.80$; Seeking Social Support: $\alpha=.86$; Active Problem Solving: $\alpha=.78$;) were administered in addition to the CSQ-CA.

\subsubsection{Statistical Analyses}

Analyses were in line with Studies 1 and 2.

\subsection{Study 3 Results}

\subsubsection{Factor Analysis}

The estimate of the fit of the covariance matrix as implied by the single-factor model was reasonably close to the observed (asymptotic) covariance matrix: RMSEA $=.07,90 \%$ CI $(.05, .09)$, comparative fit index $=.78$, even though, as expected, the single-factor model did not completely fit the observed (asymptotic) covariance matrix, adjusted $\chi^{2}(119)=189.68 ; p<.001$. All items were significantly, $p \leq .01$, related to the latent scale, except for Item $1(p=.05)$, Item $12(p=.26)$ and Item $15(p=.06)$.

\subsubsection{Reliability}

Table 2 presents the means and standard deviations of all items and the CSQ-CA total score. Reliability was good, $\alpha=.80$. Corrected item-total correlations varied between 
.15 (Item 12) and .58 (Item 14). Boys (mean = 32.24) and girls $($ mean $=33.65)$ did not differ in CSQ-CA scores, $t(111)=1.22 ; p>.05$, and age was significantly correlated with CSQ-CA scores, $r=.30 ; p<.01$.

\subsubsection{Convergent and Divergent Validity}

The CSQ-CA total score correlated positively with self-reported adolescent problems (YSR Total Problems score), $r=.62, p<.01$, but not with daily hassles (DDHQ), $r=.14 ; p=.21$. The CSQ-CA total score further correlated negatively with schoolrelated self-esteem (SPQ), $r=-.39, p<.01$, but not significantly with coping strategies and the total score (UCL-A Total Coping), $r=-.15, p=.13$. See for details of correlations with all the subscales, Table 4.

\subsection{Study 3 Conclusion}

In this last sample of adolescents with parents without chronic illnesses, the CSQ-CA also showed good reliability and the estimate of the fit of the one-factor model was reasonable. As expected, positive correlations were found between stress and internalizing and externalizing problem behaviors. A significant positive correlation with subjective experience of daily hassles was not found in this sample. In line with findings of Study 2, negative correlations were found with school-related self-esteem, but correlations with coping strategies such as seeking social support, and problem solving, were not statistically significant.

\section{Comparison of Adolescent CSQ-CA Scores across the three Studies}

Study 1 Around $12 \%(n=84)$ of the adolescents reported to suffer from (mild forms of) CMC's, of which asthma occurred most often $(n=32)$ followed by severe headaches or migraine $(n=12)$. Significant differences in perceived stress were found between adolescents with and without CMC's, $t(710)=2.73 ; p<.01$. Adolescents with a $\mathrm{CMC}$ indicated to experience more stress than adolescents without a CMC (mean CSC-Q scores were 37.19 and 34.73, respectively).

Study 2 and 3 When data from Study 2 and Study 3 were compared, significant differences in perceived chronic stress were found between adolescents with parents with and without CMC's, $t(268)=2.16 ; p=.02$. As expected, adolescents from parents with a severe CMC reported higher chronic stress levels on the CSQ-CA $(M=34.82$; $S D=8.24)$ than adolescents from parents without a CMC $(M=32.84 ; S D=6.05)$. Among the adolescents of Study 2 and Study 3 there were 28 with a mild CMC. The mean scores of the CSQ-CA for the four subgroups were: $32.99(S D=6.17 ; n=103$, healthy adolescents and healthy parents), 34.70 ( $S D=8.41 ; n=142$, healthy adolescents, parents with a severe CMC), $31.11(S D=4.28 ; n=9$, adolescents with a mild CMC, healthy parents), and $35.83(S D=6.89 ; n=19$, adolescents with a mild CMC and parents with a severe $\mathrm{CMC}$ ). In spite of the low number of adolescents with a mild $\mathrm{CMC}$, we may conclude that these data confirm the results of Study 1, in that more chronic, stressful circumstances relate to higher scores on the CSQ-CA. 


\section{Discussion}

The aim of the study presented here was to assess psychometric properties of the first questionnaire measuring feelings of chronic stress in children and adolescents. We examined the factor structure, reliability, convergent, and divergent validity of the CSQ-CA in three different adolescent samples (general population, and adolescents of chronically ill and healthy parents). Results of the three studies can be summarized along the hypotheses we tested.

First, reliability was good and factor loadings of all items were adequate in all three samples, and although the hypothesized single-factor structure did not match all data perfectly, a one-factor solution seemed to provide a reasonable fit overall. Second, the convergent validity of the CSQ-CA was supported by the positive relationship with constructs such as internalizing (i.e., anxiety and depression) and externalizing (i.e., acting out) problem behavior, experience of daily hassles, and with maladaptive emotion regulation strategies such as rumination, catastrophizing, self-blame and other-blame. These findings are in line with the results of Tolan et al. (2002), who used a social stress measure and found health problems to be related to internalizing and externalizing problem behavior and family transitions to externalizing problems in inner-city youth. Comparatively, self-reported stress predicted increases in rumination 4 months later, which in turn, mediated the relationship between self-reported stress exposure and increases in anxiety over a 7-month period in adolescents (Michl et al. 2013). Due to the paucity of studies examining feelings of chronic stress in children and adolescents in relation to developmental problems, we cannot further discuss our findings in the context of chronic stress and problem behavior. However, with respect to coping behaviors, a review shows that lower levels of internalizing and externalizing problem behavior are generally reported for engagement and problem-focused coping and higher levels for disengagement and emotion-focused coping (Compas et al. 2001). As these coping behaviors are related to chronic stress, we may assume that internalizing and externalizing problem behavior is related to chronic stress, too.

As expected, stress in adolescents was further negatively related (divergent validity) to lower self-esteem at school, quality of life and happiness. These inverse relationships have been shown in different studies before. For instance, parents of children with psychiatric disorders such as autism suffer from high rates of stress and impairments in quality of life (Nikmat et al. 2008), and children with psychiatric disorders and all the stressful consequences that follow, also show significantly lower qualities of life (Bastiaansen et al. 2004; Sawyer et al. 2002). In addition, a negative association between stress and happy feelings or positive affect have also repeatedly been shown before (e.g., Schiffrin and Nelson 2010). Further, we found that feeling more stressed was related to being less mindful, and vice versa. This has been confirmed in validation studies of child and adolescent mindfulness measures that were inversely related to the stress questionnaire for children used in this study, the CSQ-CA (De Bruin et al. 2011; De Bruin et al. 2013). From mindfulness intervention studies in adults it is further known that stress levels decrease significantly after training and health benefits increase (e.g., Carmody and Baer 2008; Grossman et al. 2004). Adaptive emotion regulation strategies such as acceptance, planning, putting things into perspective, and a positive focus were also negatively related to stress. From for instance studies assessing the effects of mindful parenting interventions, which partly focus on acceptance of oneself 
and the (condition of the) child, it is found that after training parents experience less stress and a higher quality of life (Bögels et al. 2013). The more one is able to accept the situation as it is, the less stressed one will feel about it. In line, a positive appraisal of a situation (even if it is stressful) might in turn lead to more positive thoughts, more positive feelings and therefore less stress. This is also one of the elements of the Appraisal Theory of Lazarus and Folkman (1984).

The expected negative relation between stress and coping skills was partly confirmed. Both the more internal resources such as positive self-esteem and the more external resources such as coping strategies (i.e., seeking social support) can be considered protective factors. Effects of daily or more chronic stress on mental health is likely to be less pronounced for children and adolescents with a higher level of these resources than for those with lower self-esteem and less coping skills (Dumont and Provost 1999).

Third, as expected, adolescents with a chronic illness reported higher rates of chronic stress than those without a chronic illness like asthma or migraine. In line, adolescents of parents with a chronic illness showed higher ratings of perceived stress compared to those of healthy parents, and adolescents with a chronic illness themselves and a parent with a chronic illness, showed the highest scores across the two family types. Stress is especially associated with the child's perception of parent attachment and the parent's perception of parent-child interaction (Sieh et al. 2012). From a longitudinal study on children with a parent who suffered a stroke (Sieh et al. 2010b), it can be deducted that early prediction of chronic stress in children after parental stroke may be most accurate on the basis of depressive problem behaviors in the chronically ill parent. From the stress literature in this field, it can be concluded that positive family relationships and good mental health of parents may buffer chronic stress in children and adolescents.

Several directions for future research can be proposed. First, longitudinal studies are needed to examine which stressors or stressful life events provoke enduring chronic stress. In this respect, a relevant question is whether there are specific risk or protective factors, such as personality traits, socioeconomic status, or social support. Because the stressor itself may be subject to age-related changes, Zimmer-Gembeck and Skinner (2011) emphasize to consider the stressors children are facing in the light of their coping strategies. In our view, the same notion may apply to feelings of chronic stress. Further, it would be interesting to assess intervention effects with the CSQ-CA, for instance, whether this instrument is sensitive to measure stress reduction in children and adolescents that participate in a mindfulness training.

In spite of several strengths of the current study, such as the usage of different samples, and cross-validation with a large number of variables, this study also has some limitations. One limitation concerns the generalizability of the results to adolescents in other countries. We therefore recommend estimating the reliability and validity of the CSQ-CA also in other Western and non-Western countries. In addition, in our current samples we focused on adolescents in particular, but it would be of interest to investigate whether the findings apply to the whole range of childhood to young adulthood. One psychometric study successfully used the CSQ-CA in a sample of primary school children (De Bruin et al. 2013) which seems to indicate that the CSQCA might be of value for this younger age group too. To estimate whether the CSQ-CA measures stress differently in children and adolescents, developmental aspects should be accounted for in future longitudinal studies. 
Summarizing, the current study presents the psychometric properties of the first questionnaire measuring feelings of chronic stress in children and adolescents, the CSQ-CA. Based on the good reliability, the reasonable fit of a single-factor structure, positive indications of both convergent and divergent validity in a variety of samples, the CSQ-CA appears to satisfactorily measure chronic stress in children and adolescents from 10 to 20 years. As chronic stress in children is often unnoticed and related to a wide range of problems and reduced quality of life, the CSQ-CA may be of value for clinicians monitoring the stress reducing effects of their therapeutic interventions in this age group.

Open Access This article is distributed under the terms of the Creative Commons Attribution 4.0 International License (http://creativecommons.org/licenses/by/4.0/), which permits unrestricted use, distribution, and reproduction in any medium, provided you give appropriate credit to the original author(s) and the source, provide a link to the Creative Commons license, and indicate if changes were made.

\section{References}

Achenbach, T. M. (1991). Manual for the youth self-report and 1991 profile. Burlington: University of Vermont, Department of Psychiatry.

Bastiaansen, D., Koot, H. M., Ferdinand, R. F., \& Verhulst, F. C. (2004). Quality of life in children with psychiatric disorders: self-,parent, and clinician report. Journal of the American Academy for Child \& Adolescent Psychiatry, 43, 221-230.

Bögels, S. M., Hellemans, J., Van Deursen, S., Römer, M., \& van der Meulen, R. (2013). Mindful parenting in mental health care: effects on parent and child psychopathology, parental stress, parenting, co-parenting, and marital conflict. Mindfulness. doi:10.1007/s12671-013-0209-7.

Brown, K. W., West, A. M., Loverich, T. M., \& Biegel, G. M. (2011). Assessing adolescent mindfulness: validation of an adapted mindful Attention awareness scale in adolescent normative and psychiatric populations. Psychological Assessment, 23, 1023-1033.

Byrne, D. G., Davenport, S. C., \& Mazanov, J. (2007). Profiles of adolescent stress: the development of the adolescent stress questionnaire (ASQ). Journal of Adolescence, 30, 393-416.

Carmody, J., \& Baer, R. A. (2008). Relationships between mindfulness practice and levels of mindfulness, medical and psychological symptoms and well-being in a mindfulness-based stress reduction program. Behavioral Medicine, 31, 23-33.

Compas, B. E., Connor-Smith, J. K., Saltzman, H., Thomsen, A. H., \& Wadsworth, M. E. (2001). Coping with stress during childhood and adolescence: problems, progress, and potential in theory and research. Psychological Bulletin, 127, 87-127.

De Beurs, E., van Dyck, R., Marquenie, L. A., Lange, A., \& Blonk, R. W. B. (2001). Depression, anxiety, stress scale (DASS). Gedragstherapie, 34, 35-53.

De Bruin, E. I., Zijlstra, B. J. H., van-de Weijer-Bergsma, E., \& Bögels, S. M. (2011). The mindful attention and awareness scale for adolescents (MAAS-A). Psychometric properties in a Dutch sample. Mindfulness, 2, 201-211.

De Bruin, E. I., Zijlstra, B. J., \& Bögels, S. M. (2013). The meaning of mindfulness in children and adolescents: further validation of the child and adolescent mindfulness measure (CAMM) in two independent samples from the Netherlands. Mindfulness. doi:10.1007/s12671-013-0196-8.

Dewald, J. F., Meijer, A. M., Oort, F. J., Kerkhof, G. A., \& Bögels, S. M. (2014). Adolescents' sleep in lowstress and high-stress (exam) times: a prospective quasi- experiment. Behavioral Sleep Medicine, 12, 493506.

Dufour, M. J., Meijer, A. M., van de Port, I., \& Visser-Meily, J. M. A. (2006). Daily hassles and stress in the lives of children with chronically ill parents. Netherlands Journal of Psychology, 61, 51-64.

Dumont, M., \& Provost, M. A. (1999). Resilience in adolescents: protective role of social support, coping strategies, self-esteem, and social activities on experience of stress and depression. Journal of Youth and Adolescence, 28, 343-363.

Evans, G. W., \& English, K. (2002). The environment of poverty: multiple stressor exposure, psychophysiological stress, and socioemotional adjustment. Child Development, 73, 1238-1248. 
Forehand, R., Biggar, H., \& Kotchick, B. A. (1998). Cumulative risk across family stressors: short- and longterm effects for adolescents. Journal of Abnormal Child Psychology, 26, 119-128.

Garnefski, N., Kraaij, V., \& Spinhoven, P. (2002). Manual for the use of the cognitive emotion regulation questionnaire. Leiderdorp: DATEC.

Garnefski, N., Rieffe, C., Jellesma, F., Meerum Terwogt, M., \& Kraaij, V. (2007). Cognitive emotion regulation strategies and emotional problems in early adolescents: The development of an instrument. European Child \& Adolescent Psychiatry, 16, 1-9.

Greco, L. A., Baer, R. A., \& Smith, G. T. (2011). Assessing mindfulness in children and adolescents: Development and validation of the child and adolescent mindfulness measure (CAMM). Psychological Assessment, 23, 606-614.

Grossman, P., Niemann, L., Schmidt, S., \& Walach, H. (2004). Mindfulness-based stress reduction and health benefits: a meta-analysis. Journal of Psychosomatic Research, 57, 35-43.

Hartong, I.M., Krol, M., Maaskant, A., Te Plate, A., \& Schuszler, D. (2003). Stress Vragenlijst voor Kinderen (SVK). Chronic stress questionnaire for children and adolescents (CSQ-CA). Unpublished internal report, University of Amsterdam.

Hocking, M. C., \& Lochman, J. E. (2005). Applying the transactional stress and coping model to sickle disease and insulin-dependent diabetes mellitus: identifying psychosocial variables related to adjustment and intervention. Clinical Child and Family Psychology Review, 8, 221-246.

Huizinga, G. A., Visser, A., van der Graaf, W. T. A., Hoekstra, H. J., Klip, E. C., Pras, E., et al. (2005). Stress response symptoms in adolescent and young adult children of parents diagnosed with cancer. European Journal of Cancer, 41, 288-195.

Lazarus, R. S., \& Folkman, S. (1984). Stress, appraisal, and coping (p. 1984). New York: Springer.

Lovibond, S. H., \& Lovibond, P. F. (1995). Manual for the depression anxiety stress scales (2nd ed.). Sydney: Psychology Foundation.

Lyubomirsky, S., \& Lepper, H. S. (1999). A measure of subjective happiness: preliminary reliability and construct validation. Social Indicators Research, 46, 137-155.

Meijer, A. M., van Oostveen, S. J. E., \& Stams, G. J. J. M. (2008). Zorgen voor een zieke ouder: De relatie tussen mantelzorg, ziekte van de ouder en gedragsproblemen bij kinderen [Taking care of a sick parent: the relationship between care by relatives, illness of the parent and behavior problems in children]. Kind en Adolescent, 29, 208-220.

Michl, L. C., McLaughlin, K. A., Shepherd, K., \& Nolen-Hoeksema, S. (2013). Rumination as a mechanism linking stressful life events to symptoms of depression and anxiety: longitudinal evidence in early adolescents and adults. Journal of Abnormal Psychology, 122, 339-352.

Nikmat, A. W., Ahmad, M., \& Razali, S. (2008). Stress and psychological wellbeing among parents of children with autism spectrum disorder. ASEAN Journal of Psychiatry, 9, 65-72.

Pedersen, S., \& Revenson, T. A. (2005). Parental illness, family functioning, and adolescent wellbeing: a family ecology framework to guide research. Journal of Family Psychology, 19, 404-419.

R Core Team (2012). R: A language and environment for statistical computing. Vienna: R Foundation for Statistical Computing. ISBN 3-900051-07-0, URL http:/www.R-project.org/.

Rosseel, Y. (2013). Lavaan: Latent variable analysis. R package version 0.5-14.

Sawyer, M. G., Whaites, L., Rey, J. M., Hazell, P. L., Graetz, B. W., \& Baghurst, P. (2002). Health-related quality of life of children and adolescents with mental disorders. Journal of American Academy of Child \& Adolescent Psychiatry, 41, 530-537.

Schiffrin, H. H., \& Nelson, S. K. (2010). Stressed and happy? Investigating the relationship between happiness and perceived stress. Journal of Happiness Studies, 11, 33-39.

Schreurs, P.J.G., Van de Willige, G., Tellegen, B., \& Brosschot, J.F. (1993). Utrechtse Coping Lijst: UCL-A handleiding [Utrecht coping List: UCL-A manual]. Lisse: Swets en Zeitlinger.

Sieh, D. S., Meijer, A. M., Oort, F. J., Visser-Meily, J. M. A., \& Van der Leij, D. A. V. (2010a). Problem behavior in children with a chronically ill parent: a meta-analysis. Clinical Child and Family Psychology Review, 3, 384-397.

Sieh, D. S., Meijer, A. M., \& Visser-Meily, J. M. A. (2010b). Risk factors for stress in children after parental stroke. Rehabilitation Psychology, 55, 391-397.

Sieh, D. S., Dikkers, A. L. C., Visser-Meily, J. M. A., \& Meijer, A. M. (2012). Stress in adolescents with a chronically ill parent: inspiration from Rolland's family systems-illness model. Journal of Developmental and Physical Disabilities, 6, 591-606.

Sieh, D. S., Visser-Meily, J. M. A., Oort, F. J., \& Meijer, A. M. (2014). Mediators for internalizing problem behavior in adolescents with a chronically ill parent. Journal of Developmental and Physical Disabilities, 24, 67-82. 
Tkach, C., \& Lyubomirsky, S. (2006). How do people pursue happiness? Relating personality, happinessincreasing strategies, and well-being. Journal of Happiness Studies, 7, 183-225.

Tolan, P. H., Gorman-Smith, D., Henry, D., Chung, K., \& Hunt, M. (2002). The relation of patterns of coping of inner-city youth to psychopathology symptoms. Journal of Research on Adolescence, 12, 423-449.

Van der Wolf, J.C. (1995). School perception questionnaire. Unpublished internal report, University of Amsterdam.

Varni, J. W., Seid, M., \& Rode, C. A. (1999). The PedsQLTM: measurement model for the pediatric quality of life inventory. Medical Care, 37, 126-139.

Varni, J. W., Seid, M., \& Kurtin, P. S. (2001). PedsQL(tm) 4.0: reliability and validity of the pediatric quality of life inventory (tm) version 4.0 generic core scales in healthy and patient populations. Medical Care, 39, 800-812.

Verhaeghe, S., Defloor, T., \& Grypdonck, M. (2005). Stress and coping among families of patients with traumatic brain injury: a review of the literature. Journal of Clinical Nursing, 14, 1004-1012.

Williamson, D. E., Birmaher, B., Ryan, N. D., Shiffrin, T. P., Lusky, J. A., Protopapa, J., et al. (2003). The stressful life events schedule for children and adolescents: Development and validation. Psychiatry Research, 119, 225-241.

Zimmer-Gembeck, M. J., \& Skinner, E. A. (2011). Review: The development of coping across childhood and adolescence: An integrative review and critique of research. International Journal of Behavioral Development, 35, 1-17. 